\title{
Germanica
}

\section{Identitätskonstruktionen und Essensdarstellungen in der Migrationsliteratur am Beispiel von Aglaja Veteranyis Roman Warum das Kind in der Polenta kocht und Saša Stanišićs Wie der Soldat das Grammofon repariert}

Building identities and the representation of meals in exile literature. Aglaja Veteranyi, Why the child was being cooked with polenta and Saša Stanišić, The soldier and the gramophone

Constructions identitaires et représentations du repas dans la littérature d'exil : Aglaja Veteranyi, Pourquoi l'enfant cuisait dans la polenta ?, Saša Stanišić, Le Soldat et le gramophone

\section{Sara Michel}

\section{OpenEdition} Journals

\section{Édition électronique}

URL : http://journals.openedition.org/germanica/3062

DOI : 10.4000/germanica.3062

ISSN : 2107-0784

\section{Éditeur}

Université de Lille

\section{Édition imprimée}

Date de publication : 30 décembre 2015

Pagination : 139-157

ISBN : 9782913857360

ISSN : 0984-2632

\section{Référence électronique}

Sara Michel, « Identitätskonstruktionen und Essensdarstellungen in der Migrationsliteratur am Beispiel von Aglaja Veteranyis Roman Warum das Kind in der Polenta kocht und Saša Stanišićs Wie der Soldat das Grammofon repariert », Germanica [Online], 57 | 2015, Online erschienen am: 30 Dezember 2017, abgerufen am 06 Oktober 2020. URL : http://journals.openedition.org/germanica/3062 ; DOI https://doi.org/10.4000/germanica.3062 


\section{Identitätskonstruktionen und}

Essensdarstellungen in der Migrationsliteratur am Beispiel von Aglaja Veteranyis Roman Warum
das Kind in der Polenta kocht und
Saša Stanišićs Wie der Soldat das
Grammofon repariert

Sara MICHEL Universität Luigj Gurakuqi - Albania

Der Zusammenhang zwischen Literatur und Migration erlangte in der Literaturwissenschaft vor allem seit den 80 -er Jahren des letzten Jahrhunderts besondere Aufmerksamkeit.

Die wechselseitigen Kausalitäten dieser zwei Phänomene stoßen unter den Vorzeichen postkolonialer Studien, der globalen Mobilität, der Migrationsprozesse und der Produktion einer „Literatur der Verschiedenheit"1 auf ein breiteres Interesse literatur- und kulturwissenschaftlicher Fragestellungen.

1. - Dieser Begriff ist eine Anlehnung an die Bezeichnung „Poetik der Verschiedenheit" von Werner Wintersteiner. 
Das Zusammenspiel zwischen Literatur und Migration geriet dementsprechend im Fokus literaturwissenschaftlicher Analysen, in denen bestimmte Tendenzen beobachtet und untersucht werden, deren Entwicklungslinien in einem größeren Nexus von sozialen, politischen und wirtschaftlichen Bedingungen einerseits und von neueren Perspektiven eines nun mehr überwundenen national-kulturellen literarischen Selbstverständnisses andererseits, stehen.

Die zunehmenden Annäherungen zu diesem Themenbereich werden je nach Schwerpunktsetzung einzelner Ansätze unter verschiedenen Voraussetzungen geführt und definiert. Sie umfassen sowohl äußerlich- als auch innerliterarische Bedingungen. Die Begriffe für die Gattungsbestimmungen weisen dabei auf den gesellschaftlichen Wandlel der Gesellschaft im Zuge der zunehmenden Mobilität und der damit verbundenen sozialen und kulturellen Heterogenität sowie deren Reflexion in der Literatur hin.

So stellen im deutschsprachigen literaturtheoretischen Diskurs Begriffe wie „Gastarbeiterliteratur“2, „Ausländerliteratur“3 oder „Migrantenliteratur“4 einen schillernden Gegenstand dar. Diese Begriffe sind miteinander verwandt, aber nicht deckungsgleich. Ein gemeinsames Merkmal dieser Begriffe ist der Bezug auf eingewanderte AutorInnen, deren literarisches Schaffen und Biographie deutliche Schnittmengen aufweisen, und deren Werke Dimensionen neuer Kulturkonzepte wie Inter- und Transkulturalität, Postnationalität und Hybridität erschließen. Diese Konzepte stehen wiederum als eigene Genrebezeichnungen (interkulturelle, transkulturelle, postnationale Literatur) und werden für jene literarische Texte verwendet werden, „die sich nicht so einfach in bereits vorhandene Kategorien (eines nationalkulturellen Selbstverständnisses) einordnen lassen" ${ }^{* 5}$.

2. - Der Begriff „Gastarbeiterliteratur“ steht für eine Literatur, die im Zuge der Zuwanderung ausländischer Arbeitskräfte im Deutschland der '60er Jahre entstanden ist. Die Situation der Arbeitsmigration und das Leben als Gastarbeiter. Dementsprechend wohnt diesem Begriff eine sozio-politische Perspektive inne, welche neben der Bedeutung dieser Literatur als Literaturprodukt der Betroffenen auch eines der Hauptkennzeichen der ,Gastliteratur' bildet. In der literaturwissenschaftlichen Forschung reicht die Verwendung dieses Begriffes bis in die '80er Jahre zurück.

3. - „Ausländerliteratur" entstammt einem literarischen Programm und ist sehr eng verbunden mit dem Namen von Irmgard Ackermann, der Organisatorin und Vetreterin eines literarischen Förderungsprojekts des Instituts Deutsch als Fremdsprache der Universität München. Dieser Begriff umfasst die Werke jener Autoren, die unterschiedlicher sprachlicher und ethnischer Herkunft sind und die Deutsch als Zweit-oder Fremdsprache gebrauchen.

4. - „Migrantenliteratur“ geht von einer Überbetonung der Autorenbiographie aus.

5. - Rösch, Heidi: Migrationsliteratur im interkulturellen Diskurs. Der Text basiert auf dem Vortrag zu der Tagung Wanderer - Auswanderer - Flüchtlinge 1998 an der TU Dresden. 
Ein Blick auf die Entstehung dieser Begriffe zeigt „den Kontext und die Fragestellung [...], mit der die ausgewählte Literatur analysiert [...] wird"6.

Zur Vielfalt der Begriffe, die Literatur und Migration zusammenführen, gehört auch die Bezeichnung „Migrationsliteratur“, die auf die Werke von Veteranyi und Stanišić meines Erachtens nach am ehesten zutrifft.

„Migrationsliteratur" ist in derTatein viel diskutierter Begriff. Als eine neue Form der Literatur, wirft sie - wie die anderen mit ihr verwandten Begriffe - die Frage nach den „Grenzen eines Nationalliteraturbegriffs“ auf, der ,zunehmend als unzulänglich“ erscheint?.

Das macht den Umgang mit einer Literatur der Migration und über Migration umstritten.

Als eine Literatur jenseits der nationalen Zuschreibungen, stellt die Orientierung an den Autorenbiographien, nur einen Teilbereich der Untersuchungen dar, der bei weitem nicht ausschöpfend für die Voraussetzungen und das Leistungspotential dieser Literatur ist.

„Migrationsliteratur“ mag für viele Literaturkritiker kein zufriedenstellender Begriff sein. Er beschreibt aber ein eigenes Feld der Literatur, mit eigenem gesellschaftspolitischen Kontext und ästhetisch-literarischer Gestaltung.

Das bildet die Grundlage für ein Merkmalbündel der Migrations literatur, das thematisch von Schicksalen der Flüchtlingsexistenz, von der Dialektik des Verlustes und Neufindens, von Erfahrungen mit Ortsund Sprachwechsel, von Grenzüberschreitungen schlechthin geprägt ist.

Sprachlich-stilistisch bildet diese Literatur oft ein ,ästhetisches Experimentierfeld" 8 durch das Sprachspiel mit Mustern der Erst- und Zweitsprache, durch Verfremdungen und Übertreibungen, durch Ironie und sprachliche Ohnmacht als Ausdruck des drohenden Versagens der Sprache angesichts düsterer existenzieller Lebenserfahrungen sowie durch die Verschränkung von Tragik und Komik, von oralen und schriftlichen Literaturformen, von Wirklichkeit und Fiktion. Die daraus entstehende Poetik der Migrationsliteratur generiert eine Ambivalenz von transkultureller Verbundenheit und der Bindung an den Wurzeln einer ursprünglichen Herkunft.

Die meisten dieser Merkmale finden wir auch in den Romanen „Warum das Kind in der Polenta kocht“" von Aglaja Veteranyi und „Wie der Soldat das Grammofon repariert" von Saša Stanišić.

6. - Ebd.

7. - Hoff, Karin (Hg.): Literatur der Migration-Migration der Literatur. (Texte und Untersuchungen zur Germanistik und Skandinavistik), Frankfurt, Peter Lang, 2008. S. 7.

8. - Ebd., S.8. 
In Veteranyis Erstlingsroman wird die Geschichte einer rumänischen Zirkusfamilie erzählt, die die Flucht aus der kommunistischen Diktatur Rumäniens in den Westen ergreift. Aus der Perspektive eines namenlosen Kindes, der jüngeren Tochter der Familie, wird das Leben in der Fremde geschildert. Die Familie reist mit dem Wohnmobil von Zirkus zu Zirkus, sie wohnt immer woanders, die Mutter hängt an ihren „Haaren aus Stahl“ in der Zirkuskuppel und der Vater, der den Clown spielt, trinkt und prügelt nach Belieben. Zirkuszelten, Hotels, Kinderheime und billige Varietés gehören zu den wichtigsten Lebensstationen der Protagonistin. Die Familie bricht in der Fremde auseinander. Die mit der Migration verknüpften Erwartungen für ein besseres Leben, für ökonomische und soziale Sicherheit werden nicht erfüllt. Was bleibt ist ein unbehaustes Leben und die Beziehungen zu den Verwandten im Herkunftsland, die eine Art emotionale Stabilität in der physisch und psychisch bedrohten Existenz der Figuren zu garantieren vermögen.

Der Roman „Wie der Soldat das Grammofon repariert“ des aus Bosnien stammenden und nach Deutschland emigrierten Autors Saša Stanišić, wird größtenteils ebenfalls aus der Kinderperspektive erzählt: Es ist dies der kleine schlaue Junge namens Aleksandar, der in der bosnischen Stadt Višegrad aufwächst, einen Zauberstab hat, mit dem er seinen vielgeliebten Opa Slavko wiederbeleben möchte und der ein stolzer Pionier Titos ist. Die Geschichten, die er erzählt, stammen aus der unmittelbaren Umgebung der Familie und seiner Heimatstadt, die mit dem Kriegsausbruch nicht mehr die gleiche bleibt. Die Familie flieht ins Ausland, um sich vor dem Krieg in Sicherheit zu bringen. Das ausgewählte Land ist Deutschland, in dem Onkel Bora bereits seit mehreren Jahren als Gastarbeiter lebt. Im fremden Land beginnt Aleksandar die Suche nach seiner Freundin Asija, um die er sich während des Krieges in Višegrad gekümmert hatte die Suche nach ihr ist zugleich auch Die Suche nach seiner Kindheitsheimat, nach Erinnerungen einer vergangenen Zeit, die für das gegenwärtige Ich des Protagonisten von grundlegender Bedeutung ist. Diese Suche setzt der nunmehr erwachsene Aleksandar in seinem Herkunftsland fort. Die Rückkehr in seine „Erinnerungsorte“, die in Listen mit Personen-, Straßen-, Gegenständen-, und Restaurantsnamen angefertigt waren, ist der Versuch, die unfertigen Bilder seiner Kindheit ,zu Ende zu malen.“

Beide Romane sind autobiographisch besetzt, weshalb es auf der Hand liegt, Parallelen zwischen den Protagonisten der Texte und ihren Autoren zu ziehen. So sind die Autorin Veteranyi und der Autor Stanišić selbst als Kinder mit ihren Familien in den Westen geflohen. Die Begleitumstände ihrer Migration sind denen der Protagonisten im Buch sehr ähnlich, ja fast identisch. So ist Veteranyi, wie ihre Protagonistin im Roman, auch als Zirkuskind in Bukarest geboren, mit der Familie 
nach Zürich gekommen und war bis zu ihrem fünfzehnten Lebensjahr Analphabetin. Der Autor Stanišić ist, wie seine Hauptfigur im Roman, in Višegrad geboren, hat als Kind den Krieg in Bosnien erlebt und ist 1992, im Alter von vierzehn Jahren, mit seinen Eltern nach Deutschland geflohen. Diese Anlehnung an autobiographische Momente gibt den beiden Romanen Züge ,semifiktionaler (Mikro)Biographien.“9

Der Zusammenhang zwischen der Migrationsbiographie der AutorInnen und ihren Texten ist nur insofern von Relevanz, als er meines Erachtens nach eine Produktionsbedingung darstellt, die die Stoffwahl, die wiederkehrenden Motive, deren sprachliche Aneignung und die Erzählperspektive von Migrantenprotagonisten auf gewisse Weise diktiert und prägt.

Die literarische Bearbeitung von Migrationserfahrungen, die Mehrsprachigkeit und die Vielstimmigkeit in Form und Inhalt, der Blick auf das Eigene und das Fremde, die Auseinandersetzung mit Grenzräumen und die Entstehung von hybriden Identitätsentwürfen machen diese Romane der Migrationsliteratur zugehörig.

Kulinarische Erfahrungen in „Warum das Kind in der Polenta kocht" und „Wie der Soldat das Grammofon repariert"

Die Migrationsliteratur als eine Literatur, die Fragen interkultureller Begegnungen thematisiert, bietet auch zahlreiche inhaltliche Anbindungen an kulturellen Praktiken des Essens, die in einem Verflechtungszusammenhang von Migrationserfahrungen, kulturellen Grenzziehungen und Identitätsfragen stehen.

Das Spektrum dieses Themenkomplexes wird auch in den Romanen von Veteranyi und Stanišić literarisch verarbeitet.

Essensdarstellungen gehen in diesen Texten über den rein alimentären Aspekt des Essens und Trinkens hinaus. Sie sind sowohl in individuellen als auch in einen gesellschaftlichen Rahmen, in soziale und in kulturelle Systeme eingebettet und schildern ,das von Situation zu Situation unterschiedlich gestaltete und begründete Zusichnehmen zubereiteter Speisen“10.

Die Implikation von Essen ist im Roman Veteranyis bereits im Titel enthalten. „Warum das Kind in der Polenta kocht“ ist nicht nur eine ungewöhnliche Frage. Es ist ein Bild, das den ganzen Roman durchzieht und bei dem das Kannibalismus-Motiv anklingt. Das Motiv des Kindes,

9. - Laura Gieser, «Heimatlose Weltliteratur? Zum Werk von Aglaja Veteranyi», Germanica 38, 2006, S.2.

10. - Alois Werlacher/Regina Bendix (Hg.): Kulinaristik Forschung - Lehre Praxis, Berlin, LIT, 2008. S. 3. 
das in der Polenta kocht, spielt in vieler Hinsicht eine wichtige Rolle. Es ist ein Mittel zur Gestaltung der düsteren Atmosphäre im Roman als auch zur Figurencharakterisierung der Protagonistin. Das Märchen vom Kind, das in der Polenta kocht, prägt die kindliche Erlebniswelt und vergegenwärtigt die existenzielle Rolle der Ernährung, die an Leben und Tod gebunden ist.

Dieses Bild ringt um beides und ist schlüssig für den emotionalen Anteil der Identitätsbildung der Protagonistin, die von Angst und Schmerz geprägt ist.

Diese Erfahrung gründet auf der Perspektive eines ausgewanderten Kindes, das ein Leben in der Fremde, am Rande der Gesellschaft, in einer vom Zerbrechen bedrohten Familie führt.

Durch die Erzählung über das in der Polenta kochende Kind verdrängt das andere Kind im Text die apokalyptische Vorstellung vom Tod der Mutter, die gefährlichen Auftritten im Zirkus ausgesetzt ist:

Während meine Mutter in der Kuppel an den Haaren hängt, erzählt mir meine Schwester „DAS MÄRCHEN VOM KIND, DAS IN DER POLENTA KOCHT, um mich zu beruhigen" 11 .

Die auch formal durch Großbuchstaben Hervorhebung vom „KIND IN DER POLENTA“" steht für das allgegenwärtige Gefühl von Trennung und Tod, das die Kindheit der Protagonistin charakterisiert:

Im Bett denke ich ständig daran, dass meine Mutter jetzt an den Haaren hängt. Meine Schwester muss beim KIND IN DER POLENTA immer grausamere Dinge erfinden ${ }^{12}$.

Den vom Kind vorgestellten Szenarien, warum das Kind in der Polenta kocht, ist gemeinsam, dass sie alle Verlustängste thematisieren, die durch Bilder von Essen und Kochen vermittelt werden. Diese Bereiche öffnen einen Imaginationsraum, in dem Außen- und Innenwelt auf komplexe Weise vermischt werden:

AM LIEBSTEN HABE ICH GESCHICHTEN MIT MENSCHEN, DIE ESSEN ODER GEKOCHT WERDEN ${ }^{13}$.

Diese enge Anbindung von Essen und Kochen an sinnliche und emotionale Wahrnehmung en wird nicht nur metaphorisch im Bild des Kindes in der Polenta dargestellt, sondern findet ihren Ausdruck auch in der Darstellung „banaler“kulinarischer Praxisformen wie das Einkaufen, das gemeinsame Essen oder der Umgang mit Essensüberresten.

11. - Veteranyi, Aglaja: Warum das Kind in der Polenta kocht, München, Deutscher Taschenbuch Verlag, 2008. S. 31.

12. - Ebd., S. 92.

13. - Ebd., S. 75. 
Im privaten Rahmen des Alltags inszeniert, fungiert die Familie als ein kulinarischer Ort, in dem „,der Geschmack an bestimmten Speisen und Lebensmitteln [...] vermittelt und geteilt" $" 14$ wird.

Dieser Ort wird-gemäß eines geschlechtlich kodifizierten Stereotyps - in der Figur der Mutter verkörpert. Mit ihr geht die namenlose Tochter, zugleich Protagonistin im Roman, auf den Markt einkaufen. Die Mutter entscheidet, was gekauft wird und was nicht, sie hat also die Macht, Essensvorschrift und Geschmacksorientierung in der Familie zu definieren und zu prägen. Die Auswahl der zu kaufenden Lebensmittel berücksichtigt aber auch die Vorlieben des Kindes, was bei der problematischen Beziehung Mutter-Tochter zu einem Stück Einverständnis und gegenseitig akzeptierter Angewiesenheit beiträgt:

„Beim Fischstand schaue ich den lebendigen Fischen zu, aber meine Mutter kauft fast nie Fisch, weil ich mich davor ekle. Nur selten kauft sie einen für sich und macht daraus Fischsuppe“15.

Das kulinarische Handeln der Mutter wird zu Hause weiter vollzogen. Sie bekocht die Familie und das gemeinsame Mahlritual bringt Mutter und Tochter näher durch die von Geschmacks- und Geruchssinn geprägte zwischenmenschliche Beziehung. Das Essen der Mutter vereint und stiftet das vertrauliche Gefühl des Zuhauses, das für emotionale Sicherheit sorgt.

Dieses Gefühl ist vor allem angesichts des Lebens in der Fremde von existentieller Bedeutung. Somit gewährleistet Essen nicht nur als physisch-materielle, sondern auch als psychische und seelische Versorgung die lebensnotwendige Stabilität und Kontinuität bei der Einrichtung einer vertrauten (kulinarischen) Heimat:

„Die gerösteten Auberginen meiner Mutter riechen überall wie zu Hause, egal, in welchem Land wir sind“"16.

Die eigene Essbiographie fasst das Kind in einer Liste zusammen, die aus verschiedenen Nahrungsmitteln, Zubereitungsweisen und Geschmacksrichtungen besteht. Weniger ausführlich werden die Essensvorlieben der Eltern dargestellt, die auf jeweils einen Satz reduziert werden: „Mein Vater ißt am liebsten Rühreier mit Tomaten drin“17

14. - Christine Lehmann, „Türkische Currywurst mit Pommes. Die Esskultur türkischer Migranten zwischen Narrativ und Hybridisierungen“, in: Dorothee Kimmich, Schamma Schahadat (Hg.), Essen, Bielefeld, transcript, 2012. S. 63.

15. - Veteranyi, a.a.O. S. 13.

16. - Ebd., S.10.

17. - Ebd., S. 15. 
während die Mutter am liebsten „Schwarzbrot mit Butter und Salz“18 ißt.

Die „ICH ESSE AM LIEBSTEN“19 - Liste der kindlichen Protagonistin stellt sich für den weiteren Verlauf der Geschichte als besonders wichtig heraus. Die im zweiten Teil des Romans vollzogene Dislozierung der beiden Töchter in ein Kinderheim markiert eine Zäsur, die auch die kulinarischen Genüsse des kleinen Mädchens betrifft.

Weit weg von den guten Gerüchen des Zuhauses, schmeckt das Essen hier „WIE DAS ABBAUEN DES ZIRKUSZELTES“20.

Auch der uneingeschränkte Genuss an Süßigkeiten, ein typischer Wunsch vieler Kinder, ist im Heim verboten. Die Domestizierung der Kindergelüste wird durch harte Strafen gestützt.

Alle Süßigkeiten, die uns die Eltern schicken, werden in den Schoggischrank eingesperrt. Frau Hitz entscheidet, was wir davon essen dürfen. [...] Einmal hat Frau Hitz einen Jungen erwischt, der den Schoggischrank aufknacken wollte. Zur Strafe bekam er zu jeder Mahlzeit nur Schokolade zu essen, bis er fast zusammengebrochen ist $^{21}$.

Die Bindung vom Essen an Strafen ist der Gegenpol vom Essesnerlebnis zu Hause.

Die Familie und das Kinderheim stellen im Roman die zwei wichtigsten Rahmen für die Inszenierung von Essenspraktiken. Sie gehen über die kulinarische Prägung einer im Kinderalter befundenen Protagonistin hinaus und spielen eine wesentliche Rolle bei der Weltwahrnehmung und Identitätsbildung der kindlichen Ich-Erzählerin. Auf diesen Zusammenhang wird in einem nächsten Schritt dieses Beitrags näher eingegangen.

Essensdarstellungen nehmen auch im Stanišićs Roman Wie der Soldat das Grammophon repariert einen wichtigen Platz ein. Ähnlich wie bei Veteranyi hängt das auch mit der Erzählerfigur zusammen. In beiden Texten wird die Geschichte aus der Perspektive der Hauptfiguren erzählt, die beide im Kindes- und Jugendalter sind. Das ist bekanntlich auch das Alter, in dem die kulinarische Prägung erfolgt.

Im ersten Teil des Romans, der im Unterschied zum Roman Warum das Kind in der Polenta kocht, in Herkunftsräumen ansetzt, haben wir ausführliche Schilderungen über Essenspraktiken, die in einer

\footnotetext{
18. - Ebd., S. 26.

19. - Ebd., S. 13.

20. - Ebd., S. 96.

21. - Ebd.
} 
Verschränkung von privaten und gesellschaftlichen Rahmen inszeniert werden.

Die Trauerfeier von Großvater Slavko, mit dessen Tod der Roman beginnt, führt das Private und Soziale zusammen. Es trauern nicht nur Familienmitglieder um den guten Kommunisten Slavko, sondern auch Bekannte, Freunde und Nachbarn. Sie stehen in der Küche oder sitzen im Wohnzimmer und finden Trost beim gemeinsamen Trinken und Essen.

Die männliche Trauer [...] steht in kleinen Runden in der Küche und betrinkt sich. Die weibliche Trauer sitzt mit Oma um den Wohnzimmertisch [...]. Als Opas Name fällt, schneiden die Frauen Kuchen und bieten sich gegenseitig die Stücke an. Sie zuckern den Kaffee $[\ldots]^{22}$.

Wie man dieser Szene entnehmen kann, wird die Trauer aufgrund sozialer Verpflichtungen als ein gemeinschaftsstiftender Moment beschrieben, bei dem Essen und Trinken einen integralen Bestandteil des Rituals ausmachen. Er fungiert wie ein Ausgleich für den Tod, er macht ihn erträglicher.

Eine andere Familien- und Gemeinschaftserfahrung, bei der dem Verzehr von Speisen und Getränken eine zentrale Bedeutung zukommt, ist das gesellige Zusammensein bei festlichen Anlässen. Für die Darstellung einer solchen Erfahrung liefert das Bild einer Pflaumenernte im Dorf der Urgroßeltern ein reiches Essenstableau. Hier ist ein übervoller Tisch Sinnbild für Gastfreundlichkeit und ,den konvivialen Lebensgenuss im gemeinschaftlichen Mahl“23.

Ähnlich wie bei Veteranyis Liste von Lieblingsessen, wird hier eine Art Essensmenü aufgelistet. Die Palette ist sehr breit, nicht nur was Geschmacksrichtungen, sondern auch Zubereitungsarten und Produktenvielfalt angeht. Die Darstellung vom Essensangebot bei den Urgroßeltern wird über mehrere Seiten geschildert, was die Bedeutung des Essens für ,traditionelle Formen der familiären bis gruppen-kulturellen Gastlichkeit" 24 betont.

Das festliche Gastmahl bekommt die ausführlichste kulinarische Schilderung im Text.

Nicht weniger bedeutsam sind auch Essensdarstellungen in kriegerischen Umständen. Das unmittelbare Eintauchen in den Kriegsgeschehen

22. - Stanišić, Saša: Wie der Soldat das Grammofon repariert, München, btb, 2008. S. 13.

23. - Bernhard Tschoffen, „Kulinaristik und Regionalkultur“, in: Wierlacher, a.a.O. S. 64.

24. - Regina Bendix, „Kulinaristik und Gastlichkeit aus der Sicht der Kulturanthropologie“, in: Wierlacher, a.a.O. S.46. 
wird schon im Titel des dazugehörigen Kapitels an das Motiv des Essens gebunden: „Was wir im Keller spielen, wie die Erbsen schmecken, warum die Stille ihre Zähne fletscht, wer richtig heißt, was eine Brücke aushält, warum Asija weint, wie Asija strahlt" “25.

Das Eindringen der Soldaten in das Leben der Figuren setzt ausgerechnet mit dem gewaltsamen Eindringen in den intimen, privaten Bereich des Essens ein. Der vertraute Raum wird von „ungebetenen Gästen“" gestümt.

Kaum haben die Mütter zum Abendessen gerufen, mit flüsternden Stimmen, stürmen Soldaten das Hochhaus [...]. Sie bringen eigene Löffel mit [...] Schnell zu Tisch, Soldaten, bitte, es wird doch kalt, hatten die Mütter nicht gerufen ${ }^{26}$.

Die Gewalt, mit der sich die Soldaten ans Essen heranmachen, wird an sexuelle Gewalt gekoppelt. Durch die Zusammenführung dieser zwei Bereiche wird eine Verbindung der Soldaten mit dem Animalischen suggeriert.

Und ihr! Mehr Speck in die Erbsen! So werde ich nicht satt! Ohne Speck wäre das Leben nur noch lumpig. Du da hinten, du wirst mir Speck aufschneiden - er zeigt mit dem Löffel auf Amela aus dem zweiten Stock $^{27}$.

[...] der Soldat mit dem goldenen Zahn [...] kommt aus Amelas Wohnung. [...] Hinter ihm kniet Amela mit einem nassen Schleier aus Strähnen im Gesicht. [...] Auf seinen Fingern und Knöcheln, unter den Nägeln - gelber Teig 28 .

Essensdarstellung dienen hier zur Veranschaulichung von Sexualität und Aggression durch ein geschlechtlich kodiertes Bild vom Mann als ungezügelter „Genießer“ und von der Frau als Köchin und sexuelles Objekt.

Im friedlichen Bosnien dagegen, Als alles gut war ${ }^{29}$, markieren Essensdarstellungen positive Aspekte zwischenmenschlicher Beziehungen. Sie sind Ausdruck großelterlicher Liebe und Fürsorge.

Auf der Gasflamme kocht Oma Milch auf. Ich warte immer genau zwölf Minuten und trinke warme Milch, auf der anderen Seite kocht Oma Bettlaken auf ${ }^{30}$.

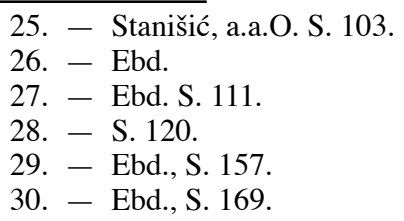


Essig-Kartoffel-Wickel senken wirklich das Fieber. Opa bringt Mandarinen und Hackfleischpflaumen ans Bett, und erklärt: das hungrige Fieber wandert in die Waden ${ }^{31}$.

Das Essmotiv fungiert in der Handlung aber auch als Zeichen für Sympathie und Aufmerksamkeit. Hier kommt dem Bekochen und Bewirten eine besondere Bedeutung zu.

Francesco zog gegenüber bei der alten Mirela zur Untermiete ein, und die alte Mirela [...] zupfte mit rosigen Wangen an den Tomaten in ihrem Garten herum. Aus dem Garten konnte man prima in Francescos Zimmer sehen. [...] Die Frauen aus unserer Straße, später auch aus der ganzen Stadt, kamen vorbei, um der alten Mirela mit dem Gras, den Karotten, den Gurken und dem Kirschbaum zu helfen [...], (S.188) Als uns die alte Mirela selbstgemachten Kirschkuchen auf die Veranda brachte, sagte Francesco zu ihr: obwohl äußerst freundlich ${ }^{32}$.

Kulinarische Erlebnisse sind auch im persönlichen Erfahrungsbereich des Protagonisten von prägender Bedeutung. So gibt es im Text genauere Beschreibungen, wie die Mutter das Brot für die Arbeit zubereitet. Wie bei Veteranyi ist auch bei Stanišić die Essenzubereitung eine weibliche Tätigkeit, wodurch kulturelle und soziale Geschlechterdifferenz erzeugt wird ${ }^{33}$.

Meine Mutter ist eine kleine Frau. Sie fährt sich durch das lange Haar, die Finger als Kamm. Sie pustet mir ins Gesicht, als würden wir spielen. Sie packt die Butter aus. Packt den Käse aus. Schmiert die Butter aufs Brot. Legt eine Scheibe Käse auf die Butter. Legt Tomaten auf den Käse. Streut zwischen Zeigefinger und Daumen Salz auf die Tomaten. Nimmt das Brot auf die Handfläche. Presst eine zweite Brotscheibe darauf. Presst fest ${ }^{34}$.

Diese Schilderung dient weniger einer Rezeptbeschreibung, wie man Brote belegt. Sie stellt vielmehr einen Kommunikationsbereich zwischen Mutter und Kind dar.

Ich sehe in ihr Gesicht, suche darin Opa Rafiks Fotogesicht. Mama siehst du Opa Rafik ähnlich?, frage ich, als sie sich an den Tisch setzt und Brot auspackt. Sie schneidet die Tomate auf. Ich warte und stelle die Frage noch einmal, jetzt erst hält Mutter inne, Messerschneide auf der Tomate 35 .

31. - Ebd., S. 187.

32. - Ebd., S. 190.

33. - Vgl. Eva Barblösius, „Weibliches und Männliches Essen rund ums Essen“, in: Wierlacher, a.a.O. S. 38.

34. - Stanišić, a.a.O. S. 22.

35. - Ebd., S. 18. 
Die besondere Wirkung des Essens im kindlichen Erlebnisraum kommt auch durch ungesunde und unbeherrschte Schlemmerei zum Ausdruck. Auf der Pflaumenernte im Dorf der Urgroßeltern ißt der kleine Aleksandar ,einen Weltrekord an Pflaumen“36. Dieser Weltrekord hat nicht einfach mit kindlicher Völlerei zu tun, sondern er steht zugleich auch für eine affektive Bindung.

Den Pflaumenhunger habe ich von meiner Mutter. Neulich, als sie sah, wie sehr ich mich über die Pflaumenernte freute, erzählte sie mir, sie habe in den letzten Monaten der Schwangerschaft nur noch Eiskunstlaufen gesehen und Unmengen an Pflaumen gegessen [...]. Ich bin meiner Mutter pflaumen- und hackfleischähnlich $[\ldots]^{37}$.

Bestimmte Essenserfahrungen werden nicht nur an bereits existierenden Bezugspersonen wie Mutter oder Großeltern gebunden, sondern sie übertragen sich auch auf Wunschvorstellungen affektiver Natur, wie im Kapitel zum Eis-Erlebnis.

Eis gibt es immer, dieses Eis gibt es nicht mehr, es ist mein Lieblingseis und hat einen Lieblingsnamen: Stela. Wenn ich eine Schwester dann habe, sage ich zu meiner Mutter und pule mit dem blauen Plastikschäufelchen im Eisbecher, dann heißt sie Stela, ja? ${ }^{38}$

Nicht zuletzt ist Essen auch bei der Ausübung vom Lieblingshobby des Kindes ein wichtiger Bestandteil. Als begeisterter Angler sitzt Aleksandar an der Drina und beginnt dem Fluss alles zu erzählen, während er seine Brote ißt.

Die Drina entlang auf dem Fahrrad [...]. Angle, fange, schwimme, rede mit dem Fluss, erzähle ihm alles, esse die Brote: Räucherschinken auf Kajmak. Pflaumenmarmelade auf Pflaumenmarmelade. So dick ${ }^{39}$.

Der Fluss ist der imaginäre Kommunikationspartner beim Essen alleine.

Seine Imaginationskraft hilft ihm auch in der Rolle vom Chefgenossen des Unfertigen. Als solcher zeichnet Aleksandar in seinen unfertigen Bildern eine schönere Welt, in der auch Wunschvorstellungen, das Essen betreffend, ihren Niederschlag finden. So widmet er ihm zwei seiner Bilder: das Bild einer „Pflaume ohne Kern im Hackfleischmantel“ und das Bild vom „Käse ohne Loch“40.

36. - Ebd., S. 33.

37. - Ebd., S. 33.

38. - Ebd., S. 165.

39. - Ebd., S. 204.

40. - Ebd., S. 301. 
Die Essensdarstellungen im Roman Stanišićs öffnen somit, ähnlich wie in Veteranyis Roman, einen Imaginationsraum, der für die kindliche Weltaneignung und Selbstwahrnehmung von grundlegender Bedeutung ist.

Identitätskonstruktionen im Kontext von Essenspraktiken in den Texten von Veteranyi und Stanišić

Die dargestellten Essensinszenierungen machen vor allem eins klar: Essen ist mehr als Essen ${ }^{41}$.

Es ist ein „soziales Totalphänomen“ (Marcel Mauss). Als solches hängt es eng mit Prozessen kultureller und sozialer Tradierungen, mit individuellen und kollektiven Identitätskonstruktionen zusammen, die auch in der literarischen Fiktion ihren Niederschlag finden.

Was sagen uns nun die oben beschriebenen Essensdarstellungen in den Texten von Veteranyi und Stanišić über diese Zusammenhänge aus?

Sowohl bei Veteranyi als auch bei Stanišić ist das Motiv des Essens von semantischer Vielschichtigkeit charakterisiert.

Ein konstitutives Element dieser Vielschichtigkeit ist der privatfamiliäre Rahmen, in dem fiktionale Essenspraktiken aus dem Alltag inszeniert werden. Hier entfaltet sich eine besondere Wirkung von Essen als Weltaneignung, in der sinnliche Erfahrungen, Emotionen, Erinnerungen und Imagination ein Identifikationsnetz projizieren.

In Veteranyis Roman identifiziert sich das Kind mit seiner Familie hauptsächlich über das Essen. Das Essen ist „Beheimatung“, das Zuhause, es ist der Raum, der am meisten die Sehnsüchte der Tochter „verkörpert“, sowohl im übertragenen wie im unmittelbaren Sinne des Wortes.

Der drohenden Entfremdung im Ausland wird mit dem Geschmack und dem Geruch vom Essen der Mutter entgegengewirkt, welches Erinnerungen an die frühere Heimat evoziert und „Bekenntnisse der Herkunft" 42 mobilisiert.

Mein Land kenne ich nur vom Riechen. Es riecht wie das Essen meiner Mutter. Mein Vater sagt, an den Geruch seines Landes erinnert man sich überall, man erkennt ihn aber nur, wenn man weit weg ist ${ }^{43}$.

Das Ausland verwandelt sich in der intimen familiären Umgebung in einer kulinarischen Heimat, die nicht so sehr an ethnische oder nationale

41. - Diese Feststellung diente Schulz als Einleitung für die von ihm herausgegebene Kulturgeschichte Essens 1995.

42. - Lehmann, a.a.O. S. 70.

43. - Veteranyi, a.a.O. S. 10. 
Identitätsmerkmalen gebunden ist, sondern an Gefühle von Vertrautem und emotioneller Sicherheit.

Die Materialität vom Essen ist für das kulinarische Erlebnis des Kindes nur zweitrangig. Anders als im Herkunftsland, wo aufgrund fehlender Nahrungsmittel Essen auf ihre Ernährungsfunktion reduziert wird, wird dem materiellen Überfluss seitens der Protagonistin wenig Beachtung geschenkt, denn Familienessen bedeutet für sie in erster Linie emotionelle Stabilität und Sehnsucht nach Geborgenheit.

Das Essen meiner Mutter riecht zwar auf der ganzen Welt, er schmeckt aber im Ausland anders, wegen der Sehnsucht. Außerdem leben wir hier wie reiche Leute, nach dem Essen können wir die Suppenknochen mit gutem Gewissen wegwerfen, während sie zu Hause für die nächste Suppe aufbewahrt werden müssen ${ }^{44}$.

\section{DAS SCHÖNSTE}

Wenn wir nach der Vorstellung zusammen essen.

Wenn meine Mutter im Bett liegt und tief schläft.

Wenn sie in der Morgendämmerung leise aufsteht, mich zudeckt und zu kochen beginnt.

Der Geruch von verbrannten Hühnerfedern ist das Zuhause ${ }^{45}$.

Das Essen der Mutter hat einen symbolischen, affektbesetzten Bezug zum Herkunftsland und zu den zurückgelassenen Verwandten.

Die Rolle der Mutter, wie man es auch dem obigen Zitat entnehmen kann, ist für die kulinarische Erfahrung des Kindes von zentraler Bedeutung. Ihre gender-spezifische Rolle als Köchin entspricht dem traditionellen Bild weiblicher Identität und konstruiert einen Teil der komplizierten Mutter-Tochter Beziehung als eine Geschichte von Abhängigkeit und Befreiung, von Fremd- und Selbstbestimmung. Diese Beziehung wird bildlich durch die Essensmotivik dargestellt, in der die Mutterfigur sowohl positiv als auch negativ besetzt ist. Als Kochkünstlerin kann sie das Gefühl von Zuhause und Geborgenheit erst möglich machen. Als Varietékünstlerin löst sie im Kind Verlustund Todesängste aus, jedes Mal wenn es daran denkt, wie Mutter bei ihren Auftritten an den Haaren hängt. Eine bildliche Gestaltung dieser Ängste ist auch das immer wiederkehrende Motiv vom Kind, das in der Polenta kocht. Dieses Bild und die dazu erfundenen Geschichten deuten auf existentielle Verunsicherung hin. Sie bieten zugleich einen

44. - Ebd., S. 12.

45. - Ebd., S. 70. 
Kompensationsraum für Selbstbestimmung und das Ausleben von „Macht- und Rachephantasien“46.

Je bedrohlicher die Ängste, umso grausamer die Geschichten, welche mit dem Eintritt ins Kinderheim, einen Übergang vom vertrauten Familienraum in ein fremdes als unheimlich empfundenes Umfeld markieren.

Meine Schwester muss beim KIND IN DER POLENTA immer grausamere Dinge erfinden.

Ich helfe ihr nach:

SCHMECKT DAS KIND WIE HÜHNERFLEISCH?

WIRD DAS KIND IN SCHEIBEN GESCHNITTEN?

WIE IST DAS, WENN DIE AUGEN PLATZEN? ${ }^{47}$

Das Kind kocht in der Polenta, weil es andere Kinder quält. Es fängt die Waisenkinder ein, bindet sie an einen Baumstamm und saugt ihnen das Fleisch von den Knochen.

Das Kind ist so dick, dass es immer Hunger hat.

Es wohnt in einem Wald voller Knochen, an denen man es von überallher knabbern hört.

Nachts deckt es sich mit Erde zu und schläft so unruhig, dass der ganze Wald zittert ${ }^{48}$.

In diesen Szenarien reflektiert sich das Unglück des Kindes im Heim. Das Essen fungiert hier, neben der Trennung von der Mutter und der strengen Disziplinordnung im Heim, als Gradmesser für dieses Unglück. Sowohl der Geschmack als auch das gezwungene Tischverhalten verwandeln das Essen hier von einem ursprünglich als Genuss empfundenen Erlebnis - man braucht sich nur die Liste mit den Lieblingsessen vergegenwärtigen - zu einem Mittel drastischer Bestrafungen.

Wir müssen jeden Tag Flocken essen, die wie Sägemehl aussehen und die mit Obst und Milch zu einem Brei vermischt werden.

Am Anfang habe ich mich geweigert.

Morgens, mittags und abends wurde mir derselbe Teller vorgesetzt. Als ich davon aß und erbrach, musste ich vom Erbrochenen essen, um mir das Stehenlassen abzugewöhnen ${ }^{49}$.

Die Nutzung vom Essen als strafendes Erziehungsmittel wird vom Kind auf die Figur der Mutter übertragen und in der Phantasie mit dem

46. - Dettmar, Ute, Angst: Lust und Schrecken in der Kinder- und Jugendliteratur, Herausgegeben vom Institut für Populäre Kulturen der Universität Zürich und dem Schweizerischen Institut für Kinder- und Jugendmedien SIKJM, Assoziiertes Institut der Universität Zürich, S. 51.

47. - Ebd., S. 92.

48. - Ebd., S. 94.

49. - Ebd., S. 96. 
Tod bestraft. Dabei bestraft sich das Kind zugleich auch selber: „DAS KIND KOCHT IN DER POLENTA, WEIL ES DER MUTTER EINE SCHERE INS GESICHT GESTECKT HAT"50.

Die enge Verbindung der Mutterfigur an bildlichen Essensinszenierungen ist im Text allgegenwärtig. Sie steht für eine weibliche, ums Verzehren kreisende Weltaneignung, in der das Essen Gegensätzliches vereint: die heimische wie auch die fremde Welt, Belohnung und Bestrafung, Gemeinschaft und Trennung. Das fließt auch im Prozess der Identitätskonstruktion vom Kind zusammen. Sie ist von Gefühlen der Heimatlosigkeit und Fremdheit, Sehnsüchten und Erinnerungen, von Lebensbejahung und -verneinung geprägt.

Diese Identität gründet auf emotionalen Selbsterfahrungen, deren Einzigartigkeit nichts mit Nation und Ethnie, auch nicht mit Kultur im engsten Sinne des Wortes zu tun hat, sondern mit Affekten, die um die Mutterfigur kreisen und durch Essensinszenierungen literarisch ihren Ausdruck finden.

Anders als in Veteranyis Roman finden wir bei Stanišić das Essen auch im gesellschaftlichen Rahmen inszeniert.

Die soziale Dimension des Essens wird im Leichenschmaus inszeniert, im Miteinander-Speisen beim Fest der Urgroßeltern in Veletovo, zu der nicht nur die Familie, sondern auch die Nachbarschaft eingeladen ist: „Zum Klofest kamen alle Nachbarn. Selbst Radovan Bunda aus dem hohen Gebirge [...]"51.

Das gemeinschaftliche Mahl entfaltet sich hier auch als ein Kulturphänomen, gebunden an Formen traditioneller Gastlichkeit, die durch ein Bild vom ,gemeinschaftlichen Genuss wohlfälliger Speisen bei einem freundschaftlich-geselligen Zusammensein" 52 vermittelt werden. Ein übervoller Tisch mit selbstgekochten regionalen Speisen und die Musik als wichtiges Festelement liefern ein archaisches, hedonistisches Bild „ungesunder Völlerei und unbeherrschter Schlemmerei“ 53 ab.

[...] es gibt Pita mit Kartoffeln, Pita mit Brennesseln, Pita mit Kürbis, es gibt Walnusskuchen und einen Schluck Rotwein für mich; es gibt keine Reihenfolge, es gibt kein Hintereinander, es gibt ständig jemanden, der sagt, er könne nicht mehr, er bekomme unmöglich einen Bissen herunter $[\ldots]^{54}$.

50. - Ebd., S. 115.

51. - Stanišić, a.a.O. S. 38.

52. - Irmela Hijiya-Kirschnereit, ,Kulinaristik als Erforschung und Lehre kultureller Identitäten und Alteritäten“, in: Wierlacher, a.a.O. S. 154.

53. - Ebd.

54. - Stanišić, a.a.O. S. 43. 
Dieses Bild findet in der Selbst- und Fremdwahrnehmung südlicher Länder eine breite Verwendung.

Im Ausland denken die Leute, dass wir hier immer feiern, sagt mein Gastarbeiteronkel. Das stimmt nicht ganz, wir müssen ja auch irgendwann das Gefeierte aufräumen. Außerdem kostet so ein Fest auch allerhand, also müssen die Eltern tagsüber arbeiten. Meinen Urgroßeltern ist für ein Fest aber tatsächlich jeder Anlass recht ${ }^{55}$.

Ein anderer soziokultureller Akt, dem „gemeinschaftsbildende Performanzqualität" 56 zukommt, ist auch das Ritual des Kaffeetrinkens. Kaffee ist mehr als nur ein Getränk, es ist eine „soziale Institution“. In der heimischen Gesellschaft des Kindes ist der Kaffee eine kollektive Konsumform sozialer Interaktion. Sie wird im Roman am besten von der Großmutterfigur tradiert. Für sie stellt Kaffee ein Grundbedürfnis nach affektiv-sozialem Austausch dar.

Wir gehen jetzt überall Kaffee trinken, sagt Oma und verlässt die Wohnung. [...] Kaffee ist für Oma nicht nur ein Getränk, Kaffee ist: die weißen Gardinen der Nachbarin in den Himmel loben, weil sie so gut gewaschen sind ${ }^{57}$.

Kaffee und Nachbarinnen gehören zum guten Leben. [...] Wie ist gut?, fragt Mutter und setzt sich auf die Bettkante. Wenn [...] Oma Katarina nie der Kaffee und die Nachbarinnen ausgehen $[\ldots]^{58}$.

Neben der kollektiven Dimension sozialer Interaktionen, die einen Teil der kulinarischen Essensinszenierungen ausmacht, kommt dem Essen auch im engen, introspektiven Rahmen persönlicher Identitätsentwicklung eine wesentliche Bedeutung zu.

Dies nimmt vor allem in der subjektiven Erfahrung von Migration eine besondere Stelle ein. Die Migration markiert eine Zäsur, die mit dem Übergang vom Vertrauten in einen unbekannten sozialen und kulturellen Raum eintritt. Durch diese Erfahrung bekommen die heimischen vertrauten Gerichte und Gerüche einen Erinnerungscharakter ${ }^{59}$.

Zugleich öffnet die Migrationserfahrung den Blick für kulturelle Bilder des Eigenen und des Fremden im Bereich des Essens.

So gibt es nicht nur verschiedene Meinungen wie man Börek zubereitet, sondern es gibt sogar auch Orte, wo es kein Börek gibt. Dies ist

55. - Ebd., S. 37.

56. - Alois Wierlacher, „Oralität und Kulturalitat von Geschmack und Genuss“, in: Wierlacher, a.a.O. S. 158.

57. - Stanišić, a.a.O. S. 263.

58. - Ebd., S. 204.

59. - Vgl. Tschoffen, a.a.O. S. 162. 
nicht wenig schockierend für ein Kind, in dessen Weltwahrnehmung Börek bis dahin ein selbstverständliches Bestandteil gewesen ist.

Die anderen Schüler aus Bosnien kopierten das Rezept und nahmen es mit nach Hause, weil sie der Meinung waren, dass Zwiebeln nicht reinmüssen und dass man Blätterteig nehmen soll. Josip und Tomislav, zwei Jungs aus Kroatien, meinten, (S. 139) bei ihnen gäbe es gar kein Börek. Kannst du dir das vorstellen, Asija? Ein börekloses Land?60

Von kulinarischen Erfahrungen im Ausland gibt es keine ausführlichen Darstellungen.

Allein im Bericht über die in die USA ausgewanderten Eltern, haben wir ein einziges Beispiel von hybrider Kulinarik: „Abends werden andere Bosnier eingeladen. Mothermade 'Cevapcici und SupermarktHamburger werden auf der Veranda gegrillt [...]"61.

Kulinarische Ortseffekte aus der Vergangenheit werden dagegen in Listen aufgezeichnet damit die Erinnerung an sie nicht ,verdampft.'

Liste der Geruchsorte. Keller: Erbseneintopf und Kohlen. Der Friedhof in Veletovo: frisch gemähtes Gras. Zorans Tante Desa: Honig. Soldaten: Eisen und Schnaps. Drina: Drina. Spajz, die Speisekammer: Sauerteig und morsches Holz - darin der Brotkasten, die Konserven, der Zucker, das Mehl, die Tüten in Tüten, die Motten, die unergründlichen Schachteln und die verrostete Mausefalle ${ }^{62}$.

Diese Geruchsorten dienen als Marker für sinnlich wahrgenommene Erfahrungen, mit einem ausgeprägten emotionalen Gehalt.

Somit bekommt Essen bzw. die Erinnerung an das „eigene“ Essen, eine stabilisierende Funktion bei der Auseinandersetzung mit dem Anderen.

In Deutsch mussten wir einen Aufsatz zum Thema „Essen, ich habe dich gern" schreiben, und ich schrieb, wie man Börek macht. Jeder las seinen Aufsatz laut vor, und als ich dran war, lachte sich die Klasse schlapp. Dazu musst du wissen, dass Essen „hrana“ bedeutet. Ich wusste das, aber da ich an der Stadt Essen gar nichts gerne habe, schrieb ich eben über das Hackfleisch und den Yufka-Teig63.

60. - Stanišić, a.a.O. S. 140.

61. - Ebd., S. 153.

62. - Ebd., S. 263.

63. - Ebd., S. 139. 
Droht dagegen Gefahr, dass die Erinnerung aussetzt, kann das eine emotionale Identitäskrise auslösen.

Asija ich erinnere mich nicht an die Birken. Es kommt mir vor, als wäre ein Aleksandar in Visegrad und in Veletovo und an der Drina geblieben, und ein anderer Aleksandar lebt in Essen $[\ldots]^{64}$.

Die hier angesprochene innere Zerrissenheit, wird durch die Unfähigkeit zum Ausdruck gebracht, sich an eine bestimmte Geschmackserfahrung zu erinnern, die in einer früheren Phase der eigenen Lebensgeschichte eine identitätsstiftende Funktion hatte.

Was passiert, wenn man sich erinnern möchte? Man geht an jene Orte zurück, die sich im Gedächtnis fest eingeprägt haben.

Die Schlussszene im Roman bietet ein signifikantes Bild dieser Rückkehr. Es ist dies die Festtafel an Opas Grab. Wie am Beginn des Romans wird die Erinnerung an den vielgeliebten Verstorbenen mit dem dazugehörenden Ritual gewürdigt: „Das Essen in Körben laufen wir zum kleinen Friedhof. Zur Seelenmesse wird zwei Mal gegessen, erklärt mir Oma, erst ohne den Toten, dann mit ihm, dazu gibt es Wein"65.

Die Festtafel ist die Inszenierung einer kulturell-religiösen Praxis, bei der durch den Akt des gemeinsamen Essens, Intimität und Nähe entsteht. Die mehrdimensionale Bedeutung des Essens wird somit auch am Ende des Textes in einem größeren Zusammenhang von Sinnlichkeit und Affekt, von Erinnerung und Sehnsucht, von Narrativität und Identität inszeniert.

Ich esse, als hätte ich seit Tagen gehungert, manchmal legt jemand etwas auf das Grab, eine Scheibe Brot mit Griebenschmalz, ich salze das Brot $[\ldots]^{66}$.

Opa, nicht alle deine Geschichten habe ich behalten, aber einige eigene habe ich aufgeschrieben und werde sie dir vorlesen, sobald der Regen aufhört 67 .

64. - Ebd., S. 140.

65. - Ebd., S. 308.

66. - Ebd., S. 310.

67. - Ebd., S. 311. 
